\title{
Blood Cardiac Troponin Increased
}

National Cancer Institute

\section{Source}

National Cancer Institute. Blood Cardiac Troponin Increased. NCI Thesaurus. Code C80518.

A laboratory test result which indicates increased levels of cardiac troponin in a biological specimen. 